\title{
Fear and Uncertainty: The Surrogacy Triad's Experience of Social Workers' Role Ambiguity
}

\author{
Ruth Walker and Liezl van Zyl
}

Philosophy Programme, School of Social Sciences, University of Waikato, Hamilton 3240, New Zealand

Correspondence to Associate Professor Liezl van Zyl, Philosophy Programme, School of Social Sciences, University of Waikato, Private Bag 3105, Hamilton 3240, New Zealand. E-mail: liezl.vanzyl@waikato.ac.nz

\begin{abstract}
In New Zealand, as in the UK, the surrogate is the legal mother of the child until parentage is transferred by the court to the intended parents. Social workers are responsible for assessing the intended parents' suitability to parent and scrutinising the arrangement. However, courts almost invariably transfer parentage, regardless of their recommendations, with the result that social workers experience a significant amount of role ambiguity. We conducted semi-structured interviews with surrogates and intended parents about their experience of surrogacy in New Zealand, focussing on the changes they would like to see in the regulatory framework, particularly with regards to legal parentage and the involvement of social workers in assessing intending parents' suitability to parent. Thematic analysis of interview transcripts showed that role ambiguity and role conflict experienced by social workers have their corollary in the experience of intended parents and surrogates, where it manifests as either fear that the adoption could be declined or uncertainty about the purpose of the social worker's involvement. Although participants recommended that their role be eliminated-along with the adoption process itself-we argue that there is a valuable role for social workers at the beginning of the process.
\end{abstract}

Keywords: Parental order reporters, role ambiguity, social workers, surrogacy, surrogacy triad

Accepted: June 2020 


\section{Introduction}

Surrogacy arrangements are increasing worldwide as they provide a unique opportunity for couples with social or medical infertility to form a family. Current legal processes in the UK and New Zealand are fundamentally similar and reflect the fact that surrogacy in these jurisdictions remains controversial and is tolerated rather than embraced as a means of family formation. Only altruistic (unpaid) surrogacy is permitted; arrangements are not legally enforceable and the surrogate and her partner are the legal parents at birth. Parentage is transferred to the intended parents through a legal mechanism. As a result, the intended parents and surrogate must interact with a number of professionals who are not usually involved in the birth of children, including social workers, lawyers and judges. Our focus in this article is on the role of social workers in surrogacy arrangements. In particular, we are interested in surrogates' and intended parents' experience of the legal processes and their encounters with social workers as these are a valuable source of information for those who design, implement and reform policy.

Because surrogacy involves a child and the question of who should raise it, in both New Zealand and the UK child welfare services have responsibility for implementing much of the process. Social workers (in New Zealand) and parental order reporters (PORs; in the UK) are required to determine the suitability of the intended parents to care for the child and are, therefore, authorised to undertake an assessment of the adequacy of their parenting skills. They must also ensure that the surrogate has given informed consent to the transfer of custody and are charged with scrutinising the surrogacy arrangement to see that the intended parents and surrogate have complied with the rules governing them (Children and Family Court Advisory and Support Service (Cafcass), 2017; Oranga Tamariki, 2018).

In both countries, there are growing numbers of international surrogacies, which are predominantly commercial. Intended parents may have obtained legal parentage in the country where the arrangement took place but in neither the UK nor the New Zealand does that establish a legal relationship between the intended parents and the child. In order to achieve that, they have to follow the same process as domestic surrogacies. Traditional surrogacies, where the surrogate is also the genetic mother, are effectively unregulated because they do not require the assistance of a fertility treatment provider. Although some use the formal process to transfer parentage, others do not. Thus, an unknown number of these occur in both jurisdictions.

Although the role of social workers and PORs can be substantial, there is relatively little research into their perspective on their function in this new means of family formation. A recent study of PORs' 
attitudes to surrogacy found a degree of role ambiguity and role conflict in nearly half the participants (Purewal et al., 2012). A different study by the same group of researchers notes various factors that make it difficult for PORs to know how to conduct their investigations. The first has to do with the 'infrequency of the work'. Because surrogacy arrangements are still relatively uncommon, surrogacy assessments form only a small part of social workers' work. Another, related, factor is the lack of adequate guidelines. A major source of role ambiguity has to do with the fact that the courts will almost certainly issue a parental order, irrespective of whether intended parents have breached the law, given that it is generally in the best interests of the baby for the intended parents to be its legal parents. This makes it difficult for PORs to know whether to look for, and report on, such breaches (Crawshaw et al., 2013).

A Swedish study by Arvidsson et al. (2018b) found that social workers are uncertain about their role, given the absence of legal guidance, and that they wrestle with ethical questions relating to informed consent as well as the sometimes conflicting interests of the surrogate and child. In another study, Arvidsson et al. (2018a) found that intended parents were affected quite profoundly by a process they experienced as protracted and unsuitable, despite social workers' efforts to make the system work for their clients. However, the Swedish system is not similar to that of the UK or New Zealand so it is difficult to make detailed comparisons.

Between 2015 and 2018, we conducted research with intended parents and surrogates in New Zealand, focusing on their experiences of the current regulatory system, including encounters with social workers. Specifically, we examined the possibility that role ambiguity and role conflict have a direct effect on the way intended parents and surrogates experience their interactions with social workers. There is no equivalent research on the experiences of intended parents in the UK, so we do not know how they feel about the process or how interactions with PORs affect them. Our results may shed some light on the effect of role ambiguity on surrogates and intended parents in the UK, given the similarities between the systems and the processes in the UK and New Zealand.

\section{Background: surrogacy in New Zealand and the UK}

There are a few minor differences between the surrogacy processes in New Zealand and the UK that are worth noting. In the UK, there is no prior approval process for the surrogacy arrangement beyond the basic consideration of child welfare required of fertility treatment providers before they agree to treatment (Horsey, 2016). In New Zealand, in contrast, there is a dedicated ethics committee that approves all gestational surrogacy arrangements. Unlike traditional surrogacy, gestational surrogacy involves the use of either the intended mother's or the donor's eggs 
and, therefore, necessitates the use of in vitro fertilisation (IVF). Fertility clinics must have ethics approval for each arrangement before they can provide services (ACART, 2013). Furthermore, where the UK uses parental orders, issued within six months of the birth and specifically designed for surrogacy as a replacement for adoption, New Zealand uses its adoption legislation, which does not recognise surrogacy as a distinctive type of family formation. However, parental orders and adoption are functionally equivalent. They both secure legal parentage for the intended parents. The two systems share the same basic principles, and their substantive elements make them experientially similar for intended parents and surrogates. Specifically, the rules about legal parentage and the involvement of social workers in the process of transferring it leave open the possibility that, even with the consent of the surrogate, the court could decline to make the order and the intended parents be denied parentage of the child.

Unlike PORs in the UK, social workers in New Zealand are involved in surrogacy arrangements twice. The first time is to provide a letter stating the intended parents' 'potential suitability to adopt', which is a condition of obtaining ethical approval. For this, they undertake a preliminary assessment. The approval process is independent of the transfer of parentage once the baby is born. To facilitate the transfer, social workers undertake a full assessment after the surrogate becomes pregnant. Although the social workers come from the same state service, the preliminary assessment is carried out for the ethics committee and the full assessment is for the family court. Social workers have a slightly larger role than PORs because of the approval process but both are charged with the same task, which is to examine the surrogacy arrangement for compliance and to assess whether the child's welfare and interests would be served by transferring legal parentage to the intended parents.

From the perspective of the social workers and PORs, both systems operate with similar limitations. There are provisions in the legislation that generate uncertainty for them as they attempt to carry out their assigned role. The mandatory elements of the parental order regime include not only that compensation for the surrogate is barred but also that the intended parents apply within six months. Technically, an order cannot be made if these requirements are not met. The situation is New Zealand is very similar, except that the intended parents also have to go through the process of formally adopting a child from a woman who became pregnant with the sole intention of allowing them to have their own child. This makes the situation awkward for social workers who have to work with their criteria.

The most salient similarity for the social workers and PORs is that they have good reason to believe that the court will almost certainly approve the adoption or grant the order. The paramountcy principle, 
operative in the UK since 2010, means that it will almost never be in the child's best interests for the parental order to be declined. Breaches of the rules committed by the intended parents are unlikely to affect the outcome. As Horsey (2016) argues, paramountcy means there can be no balance of interests against the value of upholding public policy. Similarly, the approval process in New Zealand makes it highly unlikely that adoption orders will be declined.

As noted earlier, Crawshaw et al. (2013)'s participants felt that the 'limited formal guidance and case law' as well as the 'infrequency of the work' made it difficult for PORs to know how to conduct their investigations. To an extent, these are problems shared by New Zealand social workers. Detailed processes and documentation on adoption are available, but these provide very little guidance in the case of adoptions involving surrogacy. Formal guidance on surrogacy is sparse. For example, the key information for IVF surrogacy adoptions states that '[s]ocial workers should keep in mind that the proposal is for parents to raise their own children often carried to birth on their behalf by a close relative or friend. With this in mind, [they should] tailor the assessment accordingly looking at the six core needs of a child' (Oranga Tamariki, 2018). However, when they turn to the core needs information, there is nothing about how to apply them in a surrogacy adoption. The statement itself, while an accurate description of the reality, directly contradicts the legal facts, which are that the baby is not their own child and the surrogate is not acting on their behalf.

Professional discretion makes a substantial contribution to PORs' assessments in the absence of specific instructions, notably in the matter of reasonable expenses. There is no definitive list of acceptable expenses, and opinions vary widely over whether to inquire into them or not and over what amount of money might be reasonable. This means that if the intended parents and surrogate are assigned, a POR who asks them to provide a line-by-line accounting of their expenditure and proof that their accounts match, they will have a very different experience from the ones assigned a POR who thinks there is no point in investigating and makes only perfunctory inquiries. Yet, both can justify their approach. The former approach is taken by PORs who do not think it is their job to determine what is reasonable but to report the details so that the judge can make a determination. Even if it makes no difference to the ruling, judges have expressed disapproval of breaches. The latter approach is taken by PORs who give more weight to the fact that the order will be granted regardless of what the intended parents have paid to the surrogate and consider an investigation pointless or intrusive (Crawshaw et al., 2013, p. 1232).

Purewal et al. (2012) found that nearly half of their POR respondents were high in role ambiguity and role conflict. Those that were had a significantly less positive attitude to surrogacy itself as well as to the 
surrogates and the parental preparation of the intended parents. The researchers worry that these biases could find their way into the reports and influence the court. We think the reports could certainly colour the judge's view of the intended parents and surrogate but it is clear that they could not change the outcome unless there were grave concerns about the welfare of the child. We think, rather, that role ambiguity, role conflict and bias are more likely to have a direct effect on the interactions between social workers and intended parents and surrogates. Our data suggest that the factors identified in PORs in the two UK studies (Purewal et al., 2012; Crawshaw et al., 2013) will have their corollary in the experience of intended parents and surrogates in New Zealand.

\section{Method}

The qualitative data for this research are drawn from a study of surrogacy in New Zealand that sought to explore the experiences of intended parents and surrogates, particularly their perceptions of the current regulatory framework and how it affected them. From 2015 to 2017, the authors conducted interviews with four surrogates and four intended parents, including one heterosexual couple interviewed together, none of whom were members of the same arrangement. Thus, there were eight surrogacy arrangements comprising seven heterosexual couples and one gay couple. They included three traditional and five gestational surrogacies. Seven were domestic and one international.

\section{Participants}

Two participants, both intended parents, contacted the authors following the media interviews they had given. After ethical approval for the study was granted by the University of Waikato Human Research Ethics Committee, these contacts were sent a formal invitation to participate. The information and invitation were also posted on a closed surrogacy forum by its moderators and an unmoderated social media forum. The other six participants responded to those invitations. Eight participants were women. The intended father in the couple interviewed together was the only male. They reported on surrogacies that had occurred over a ten-year period during which there were no changes to the regulatory framework. The focus of the research is on what changes users of the system would like to see to the regulatory framework. Our sample, while not necessarily representative, is illustrative of the most serious issues surrogacy triads have with the system. 


\section{Procedure}

Participants were sent an interview schedule prior to the interview. They were free to talk about anything they wished in addition to or instead of the questions on the schedule. After obtaining written informed consent, the authors conducted semi-structured interviews ranging in length from forty-seven to seventy-seven minutes over Skype, which were recorded and then transcribed. All participants answered all of the questions and added details about aspects of the experience they felt were important. They were sent their transcripts to edit as they wished and the finalised transcript is the sole source of data for each participant.

\section{Analysis}

The finalised transcripts were imported into NVivo 11 for thematic analysis. An experienced research assistant carried out the initial coding, which was data-driven and inductive (Braun and Clarke, 2006). That is, coding did not use the interview questions as the framework. One of the authors reread all the transcripts and reviewed the coded extracts. It appeared that all participants reported interacting with professionals whom they would not encounter in a standard pregnancy or with whom they would have interacted differently. The author searched all the data extracts that were coded for contact with professionals and then reread the transcripts for the context of the interaction to determine how important they were to participants' experiences. They showed a pattern indicating that participants had to find their own way through the adoption process to a successful outcome because there were few guidelines to follow. These reports could be grouped under the theme 'navigating interactions with professionals'. All participants reported some positive and some negative experiences, so further analysis was done to identify subthemes that captured the emotions associated with their encounters. In this article, we report on the subtheme 'fear and uncertainty' as it relates to social worker involvement. All our participants reported experiences that were to a greater or lesser degree negative. We report data from five surrogacy arrangements that involved contact with social workers. The numbers assigned to the interviewees are the ones used throughout the project.

\section{Findings}

The fear most commonly associated with surrogacy is that the surrogate will decide not to relinquish the baby. High-profile cases suggest that this happens frequently although it is in fact very rare. For example, in 
the case of Baby M in 1985, US surrogate Mary Beth Whitehead went so far as to go on the run with the baby rather than relinquish her to the intended parents. A bitter and protracted legal battle ended with Baby $\mathrm{M}$ in the custody of the intended parents but still required to maintain contact with the surrogate. The case had a profound and detrimental effect on surrogacy regulation in spite of it being anomalous (Walker and Van Zyl, 2017, pp. 167-72).

As in the vast majority of surrogacy arrangements, our participants did not have to contend with any uncertainty over relinquishment. The surrogates never wavered in their view that the baby was not theirs and the intended parents had complete confidence in their surrogates. It was in their encounters with the social workers that, for some, a different fear arose that the application to adopt would be declined if they breached the rules about the payment to the surrogate or if the social worker did not deem them to be suitable parents. Yet, in reality, there was even less chance of the adoption being turned down than of the surrogate reneging on her promise to relinquish the baby. It appears to depend on the social worker whether that fear arises or not.

There is a significant mismatch between what the social worker knows (that the adoption will be approved) and what intended parents and surrogates come to believe (that it is genuinely in doubt). For S1 the message conveyed, whether intentionally or not, was somewhat intimidating:

that was an example of almost fear mongering at this first visit. 'You had better do as I say else you're not going to get this adoption going through!' and I was horrified. [S1]

Fear centred predominantly on the question of reasonable expenses, which are not clearly set out in information for intended parents. IP1 expressed just such a fear:

I know people do pay their surrogates under the table and stuff but I was like 'Jesus, do you really want to risk your adoption of your baby?' It's what you put at risk.

They made it seem like a pretty real consequence. I don't know if they would do that, but they made it seem like a pretty real consequence. The CYFS [Child, Youth and Family Services] woman was very, she was a little scary on that point, so we were like 'no, no, we've been good'. [IP1]

Participants were also aware of the variation in social workers' attitudes towards surrogacy.

You're not dealing with CYFS as a whole, you're dealing with a person who has their own opinion on adoption and surrogacy and you've got to tailor what you say to them to that person's thinking and that's what I don't like. [S1] 
The social worker's opinion could influence something as significant and sensitive as when the baby could transfer to the care of the intended parents. Under the New Zealand Adoption Act (1955), a woman cannot consent to an adoption until at least ten days after birth, which, because they are treated as working days, effectively means that the baby should stay in the care of the surrogate for twelve days. It is possible to apply for permission to transfer the baby earlier than that, but whether to provide placement approval is at the social worker's discretion and may be influenced by her beliefs. It is still widely believed that a woman will find it more difficult to relinquish a baby that is genetically hers than one that is not.

Often now CYFS see it as acceptable to give pre-birth approval for [gestational surrogacy] babies to go straight home with IPs, but this is rarely given with [traditional surrogacy] babies. Most often they are told it's the 12 day thing like a normal adoption. Once again, it depends on your CYFS worker and their opinions. [S1]

An encounter with a social worker on the telephone during her pregnancy contributed to S3's decision not to follow the formal route with her traditional surrogacy. She had not told the social worker that she was already pregnant but indicated that she was considering it.

\begin{abstract}
She went on, this was the surrogacy advocate, 'why would you get pregnant with a child who's going to lay in your womb and listen to your heartbeat and then you're going to rip them from your arms and give it to some stranger?', and I was like 'wow, I don't think we'll be needing your services, thank you for your time'.
\end{abstract}

Her decision could have caused significant legal complications but fortunately, these were resolved.

Some social workers were supportive of surrogacy and acknowledged the awkwardness of the situation they and the intended parents were in during assessments.

even the CYFS woman that came and did it was like, 'I'm sorry this is intrusive and unnecessary but it's what we do'. [IP1]

However, this was the same social worker who was 'a little scary' on the subject of payments to the surrogate so the fear persisted that anything they might do to thank the surrogate could jeopardise the adoption.

A positive experience with a sympathetic social worker was doubleedged. It raised the question of why they were required to go through a process that appeared to be pointless. This was not expressed as anger towards the social worker but towards the system. IP3 reported:

The CYFS lady that showed up at my house, she was great, I think she knew the futility of the whole thing, she showed up and had a cup of tea 
and we talked about [the town they lived in] and what a nice day it was and then she left. [IP3]

But she commented later in the interview:

That's a waste of resources for them and it's an invasion of privacy for us as well. We're not adopting a child, this is our own genetic child. After you've been through all of this and then to have to have your house looked over and have police checks... The less of that stuff the better, it doesn't serve a purpose.

IP4 had three visits from the social worker, whom she knew slightly and liked. In contrast to the other participants, IP4 was the only one who did not experience a fear that the adoption could be in doubt.

Commenting on the later visits, IP4 acknowledged the social worker's discomfort ('she did feel awkward as well') and expressed her frustration.

It felt like an exercise ticking the boxes, like there wasn't any substance. [Social Worker] was doing it because she had to, she came and apologized the whole way through it.

A social worker who clearly feels she should not be doing what she is doing cannot demonstrate the value of carrying out an assessment. IP4 was left angry on a personal level at having to justify herself as a parent and at the waste of resources.

The worst part was going through CYFS to be accepted as parents...That was the biggest insult, at the end of the day, I was just just crazy. Trying to justify ourselves as parents yes, I don't know. It was a waste of resources. And the shame of it was having to be A okayed by CYFS,

Our participants experienced two types of uncertainty, both of which appear to be in response to the messages, intentional or not, conveyed by their social workers. The first is the uncertainty of outcome, which engendered fear. They came to believe that their social workers could prevent the adoption and that compliance or non-compliance with the rules could determine whether or not they became legal parents to their baby.

Sympathetic social workers gave rise to the second form of uncertainty. They were unable to demonstrate the value of their role in the process the intended parents had to follow. If they thought it 'unnecessary' and apologised for having to carry out the assessment, then the intended parents could not be expected to see their involvement as a constructive contribution to a successful outcome. We now examine whether social workers' role ambiguity and role conflict could have played a part in the experiences our participants report. 


\section{Discussion}

Role ambiguity occurs when the behavioural expectations for fulfilling that role are unclear, vague or imprecise (Tubre and Collins, 2000; Tummers et al., 2012). A person may have a task to perform but what they are to do to accomplish that task is unclear. For example, PORs are given the task of investigating the financial arrangement between intended parents and surrogate but what this entails is unclear both in terms of role-is it a social worker's role to determine what counts as reasonable expenses or to report in detail so that the judge can determine it?-and what counts as accomplishing it or failing to accomplish it. What they do in this matter makes no difference to the outcome. Role conflict occurs when there are incompatible demands within roles (Tummers et al., 2012). For example, because New Zealand uses its adoption legislation for surrogacy, there are conflicting demands for social workers who are instructed to 'bear in mind' that the intended parents are applying to adopt their 'own' children whom another woman is bearing 'on their behalf' (Oranga Tamariki, 2018. Emphasis added). But their behaviour is also expected to conform to the process where the intended parents are adoptive parents who have 'no prior tie' to the child and the surrogate is an independent birth mother making a decision about whether to relinquish 'her' child for adoption. Tummers et al. (2012) add further refinement to the concept that allows us to specify the type of conflict involved, which is 'policy-client role conflict', which occurs when 'professionals perceive that the behaviour demanded by the policy they have to implement ... is incompatible with the behaviour demanded by their clients' (Tummers et al., 2012, p. 1042).

We can see a policy-client conflict at work if we think of intended parents and surrogates as clients. They do not see surrogacy as a form of adoption and expect the mechanism used to transfer parentage to be a formality that is minimally burdensome when both parties consent. That is their expectation of the policy. Hence, in their view, if they have received approval from the national ethics committee and their circumstances have not materially changed, they can see no reason why they should be as closely scrutinised as traditional adoptive parents. Indeed, the fact that adoption orders are almost always granted in surrogacy arrangements, regardless of breaches of the rules, supports their inference. However, the official policy derived from the legislation denies that this is the case and introduces an element of specious uncertainty, which, as we have seen, can be fear-provoking for intended parents and surrogates, and an apparent source of discomfort for social workers. Nevertheless, it is the policy they are required to implement in their role as social workers. 
Although there are subtle differences between the New Zealand system and the Parental Order regime in the UK, PORs, like their New Zealand counterparts, exhibit role ambiguity around the assessment of intended parents' parenting skills (Purewal et al., 2012; Crawshaw et al., 2013). We think it is reasonable for PORs to assess the intended parents' suitability to parent the baby, given that the UK does not have a prior approval system for surrogacies beyond the basic child welfare consideration that fertility clinics are supposed to give before accepting patients for treatment. However, the timing of the assessment is problematic and could cause role ambiguity. PORs carry out the evaluation after the baby has been placed with the intended parents. At this stage, the surrogate has already consented to the transfer of care to the intended parents, any financial transactions have already occurred, and the baby could even be well over six-month old. It is a 'fait accompli', as PORs in the studies cited above report. It is difficult for them to know what they are there for as the final outcome is not in doubt by this stage.

We suggest that intended parents and surrogates directly experience role ambiguity through the social worker's behaviour. They may come to fear (mistakenly) that the adoption could be declined if they are not extremely careful. Alternatively, they might realise that such an outcome is not a real possibility, but this generates its own uncertainty about why the social worker is even involved in the process. Our results also suggest that when PORs investigate financial arrangements in detail, intended parents are unlikely to realise that the outcome is not affected by their conduct. The process appears to have weighty consequences for the wrong answers. Only individual PORs can alleviate their concerns if they wish to do so. Ambiguity may be inevitable in a system where almost nothing the POR reports will change the foreseen outcome that the intended parents will be awarded legal parenthood. A system that generates role ambiguity and role conflict for the professionals as well as uncertainty and fear for the clients is clearly undesirable.

At the heart of policy-client role, conflict is the way social workers and the triad perceive the purpose of the process. In legal or policy terms, the processes 'establish' legal parentage through a transfer of parental rights from the surrogate who automatically has them (whether she wants them or not) to the intended parents who will raise the child. If we look at it from the perspective of the intended parents and surrogate, the clients, it would be more accurate to say that the processes 'confirm' the already existing parenthood of the intended parents. The surrogate has never regarded the baby as her baby. Her presumptive motherhood is an anachronistic burden to her. Surrogates consistently report they do not have maternal feelings towards the baby (Jadva et al., 2015; Teman and Berend, 2018). Indeed, the women in our sample 
prefer the term 'surrogate' to 'surrogate mother' precisely because they do not see themselves as any sort of mother to the baby.

Our participants-who had been assessed twice, sometimes by the same social worker-could see no meaningful role for social workers and it is difficult to see how the second, full assessment of their suitability to parent could be justified. They want social workers removed from the formalities around transfer of parentage. From their perspective, the only function social workers appear to have is to make a full assessment of their suitability to adopt their own genetic child, whose best interests will almost certainly be served by that outcome alone. Just a few months earlier, they have had a favourable preliminary assessment. Without it, the surrogacy arrangement would not be approved. Their frustration and resentment is warranted, and according to some of our participants, it is shared by their social workers as well.

The preliminary assessment shows that there is a valuable role for social workers at the beginning of the process when the triad applies for approval of their arrangement. Before the ethics committee approves an application, there must be satisfactory legal, medical and counselling reports as well as approval in principle of the intended parents' suitability to adopt any child resulting from treatment. Most applicants are approved as potential adoptive parents. In the few cases where approval was refused, it was because of serious issues that social workers uncovered in the preliminary assessment. Where there are grave concerns for the child's welfare such that a child might be removed from the care of those parents, then the time to discover them is prior to any pregnancy being established. This is an important task for social workers, and one that need not generate role ambiguity or conflict because their actions do have an effect on the outcome. Social workers do need to take more pains to communicate the value of the assessment to intended parents, but this would be much easier for them to do if the preliminary assessment was the only one they had to undertake.

We do not expect that such changes would entirely remove the fear and uncertainty experienced by intended parents in New Zealand. Although fear and uncertainty would be reduced because the social workers would not be suffering role ambiguity, an element of policyclient role conflict would remain. This is because at the heart of our participants' experience is bafflement over why they are not automatically the legal parents of any child born through the surrogacy arrangement. They had to adopt their own child and be approved to do so. Without substantial changes to the policy, which would include a change to the presumption of parenthood, the role conflict cannot be resolved; however, hard social workers try to make the process meaningful and useful to the triad.

It is interesting to note that in its joint consultation document, Building Families through Surrogacy: A New Law (Law Commission and 
Scottish Law Commission, 2019), the Welsh and Scottish Law Commissions propose a new pathway to parenthood for all domestic surrogacy arrangements. This would require clinics to carry out a preconception welfare check, but there will be no post-birth child welfare assessment of the intended parents. This would eliminate the role of PORs in domestic arrangements. However, PORs would still be involved in international surrogacy arrangements, which would follow the existing parental order pathway. We think it would be a mistake to remove trained social workers from the process entirely as they are best qualified to do safeguarding assessments. Creating a role for them at the beginning of the process, along the lines of practice in New Zealand, would remove role ambiguity and role conflict while retaining their expertise.

\section{Limitations and future directions}

We have put forward an interpretation of our results in terms of role ambiguity and role conflict, following up a question raised by Purewal et al. (2012) and claimed that the perspectives offered by our participants could be relevant to the work of PORs in the UK by showing how interactions with social workers are experienced by intended parents and surrogates. Our sample is small and although New Zealand and the UK are similar in many ways, a study of intended parents and surrogates in the UK would be needed to confirm our findings. A study of social workers in New Zealand to parallel those of Purewal et al. (2012) and Crawshaw et al. (2013) would also be valuable. It is the social work profession that bears the brunt of ill-designed policies that they have to implement. As we saw with Arvidsson et al. (2018a, b) as well as our own study, it is individual social workers who are the face of a system that seems to be wilfully denying the reality of the relationships in surrogacy and forcing people into categories they do not belong to. Change that is informed by the experiences of members of surrogacy arrangements and the social workers who interact with them is overdue.

\section{Conclusion}

Our study of surrogacy in New Zealand suggests that role ambiguity and role conflict experienced by social workers have their corollary in the experience of intended parents and surrogates, where it manifests as either fear that the adoption could be declined if they are not extremely careful or uncertainty about the purpose of the social worker's involvement. Further research with intended parents and surrogates in the UK and social workers in New Zealand would be needed to confirm our findings. However, if we are right, then policy and law should be 
reformed to provide a process that gives clarity to the role of PORs and social workers and that accurately captures the distinctive nature of surrogacy arrangements. The surrogate is not the mother of the baby and enters the arrangement on that basis. She acts on behalf of the intended parents. With the heavy demands on social workers in both jurisdictions, recognition of this unique relationship would be beneficial to the profession as well as participants in surrogacy arrangements.

\section{Acknowledgements}

This research project was funded by a Contestable Research Grant (EO57), which was awarded by the Faculty of Arts and Social Sciences at the University of Waikato, New Zealand (2015-17). We are grateful for their support, as well as to the people who participated in the research project.

\section{References}

Advisory Committee on Assisted Reproductive Technology (ACART) (2013) 'Guidelines on surrogacy arrangements involving assisted reproductive procedures', available online at: https://acart.health.govt.nz/ (accessed July 13, 2020).

Arvidsson, A., Johnsdotter, S., Emmelin, M. and Essén, B. (2018a) 'Being questioned as parents: An interview study with Swedish commissioning parents using transnational surrogacy', Reproductive Biomedicine and Society, 8, pp. 23-31.

Arvidsson, A., Johnsdotter, S., Emmelin, M. and Essén, B. (2018b) 'Gauging the interests of birth mother and child: A qualitative study of Swedish social workers' experiences of transnational gestational surrogacy', European Journal of Social Work, 21(1), pp. 86-99.

Braun, V. and Clarke, V. (2006) 'Using thematic analysis in psychology', Qualitative Research in Psychology, 3(2), pp. 77-101.

Children and Family Court Advisory and Support Service (Cafcass). 2017. 'Resources for Parents and Carers', available at: https://www.cafcass.gov.uk/grown-ups/parentsand-carers/resources-parents-carers/ (accessed July 13, 2020).

Crawshaw, M., Purewal, S. and van den Akker, O. (2013) 'Working at the margins: The views and experiences of court social workers on parental orders work in surrogacy arrangements', British Journal of Social Work, 43(6), pp. 1225-43.

Horsey, K. (2016) 'Fraying at the edges: UK surrogacy law in 2015: H v S (Surrogacy Agreement) [2015] EWFC 36, Re B v C (Surrogacy: Adoption) [2015] EWFC 17, Re Z (A Child: Human Fertilisation and Embryology Act: Parental Order) [2015] EWFC 73, A \& B (Children) (Surrogacy: Parental Orders: Time Limits) [2015] EWHC 911 (Fam)', Medical Law Review, 24(4), pp. 608-21.

Jadva, V., Imrie, S. and Golombok, S. (2015) 'Surrogate mothers 10 years on: A longitudinal study of psychological well-being and relationships with the parents and child', Human Reproduction, 30(2), pp. 373-9. 
Law Commission and Scottish Law Commission. (2019) Building Families Through Surrogacy: A New Law, available online at: https://www.lawcom.gov.uk/project/sur rogacy/ (accessed July 13, 2020).

Oranga Tamariki. 2018. 'Surrogacy and Adoption', Available at: https://www.orangata mariki.govt.nz/adoption/surrogacy-and-adoption/ (accessed July 13, 2020).

Purewal, S., Crawshaw, M. and van den Akker, O. (2012) 'Completing the surrogate motherhood process: Parental order reporters' attitudes towards surrogacy arrangements, role ambiguity and role conflict', Human Fertility, 15 (2), pp. 94-9.

Teman, E. and Berend, Z. (2018) 'Surrogate non-motherhood: Israeli and US surrogates speak about kinship and parenthood', Anthropology \& Medicine, 25(3), pp. 296-310.

Tubre, T. C. and Collins, J. M. (2000) 'Jackson and Schuler (1985) revisited: A meta-analysis of the relationships between role ambiguity, role conflict, and job performance', Journal of Management, 26(1), pp. 155-69.

Tummers, L., Vermeeren, B., Steijn, B. and Bekkers, V. (2012) 'Public professionals and policy implementation', Public Management Review, 14(8), pp. 1041-59.

Walker, R. and Van Zyl, L. (2017) Towards a Professional Model for Surrogate Motherhood, London, Springer. 